\title{
LETTER
}

Transcriptional control and signal transduction

\section{EZH2 inactivation in RAS-driven myeloid neoplasms hyperactivates RAS-signaling and increases MEK inhibitor sensitivity}

\author{
Johannes Lorenz Berg ${ }^{1}$ - Bianca Perfler ${ }^{1}$ - Stefan Hatzl $\mathbb{1}^{1} \cdot$ Barbara Uhl $^{1} \cdot$ Andreas Reinisch $^{1} \cdot$ Gudrun Pregartner $^{2}$. \\ Andrea Berghold ${ }^{2}$ - Thomas Penz ${ }^{3}$ - Michael Schuster (D) $^{3}$ • Klaus Geissler (iD ${ }^{4,5}$ - Andreas Prokesch ${ }^{6,7,8}$.

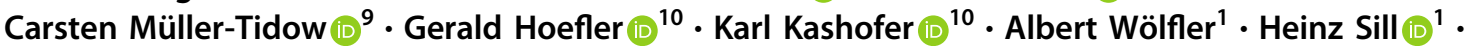 \\ Veronica Caraffini $\mathbb{D}^{1,11} \cdot$ Armin Zebisch ${ }^{1,12}$
}

Received: 17 August 2020 / Revised: 11 January 2021 / Accepted: 26 January 2021 / Published online: 15 February 2021

(c) The Author(s) 2021. This article is published with open access

\section{To the Editor}

Mutations modifying $R A S$ (RAS ${ }^{m u t}$ ) occur frequently in myeloid neoplasms $(\mathrm{MN})$ and play a key role in myeloid leukemogenesis [1, 2]. The most commonly observed $R A S^{m u t}$ in MN comprise aberrations in NRAS and KRAS, as well as in three genes that modulate the levels of active RAS-GTP (NF1, PTPN11, and CBL) [3]. Mechanistically, $R A S^{m u t}$ activate a multitude of downstream signaling cascades, with the MAPK/ERK module being considered one of the major RAS-effector pathways [4]. Consequently, pharmacologic MAPK/ERK inhibition-i.e., by MEK inhibitors - is an appealing therapeutic approach. Indeed, the development of MN in Ras $^{m u t}$ mice can be effectively attenuated by treatment with these substances [2].

These authors contributed equally: Veronica Caraffini, Armin Zebisch

Supplementary information The online version contains supplementary material available at https://doi.org/10.1038/s41375021-01161-0.

Veronica Caraffini

vc390@mrc-cu.cam.ac.uk

$\triangle$ Armin Zebisch

armin.zebisch@medunigraz.at

1 Division of Hematology, Medical University of Graz, Graz, Austria

2 Institute for Medical Informatics, Statistics and Documentation, Medical University of Graz, Graz, Austria

3 CeMM Research Center for Molecular Medicine of the Austrian Academy of Sciences, Vienna, Austria

4 5th Medical Department with Hematology, Oncology and Palliative Medicine, Hospital Hietzing, Vienna, Austria

5 Sigmund Freud University, Vienna, Austria
Unfortunately, these promising results could not be translated into human $\mathrm{MN}$, with disappointing results in clinical trials [5]. One potential reason is the fact that $R A S^{\text {mut }}$ do not exist as solitary events within these tumors $[1,6]$. The existence of co-occurring mutational and non-mutational aberrations has the potential to further influence the activating effects of $R A S^{\text {mut }}$, which ultimately aggravates or inhibits $R A S^{m u t}$-driven leukemogenesis and thereby changes the dependency on activated RAS-signaling [6, 7]. Consequently, these co-occurring events might also change the sensitivity to MEK inhibitors, as recently shown for the coexistence of mutations in NRAS and TET2 [7]. Enhancer of zeste homolog $2(\mathrm{EZH} 2)$ is the core component of the Polycomb Repressive Complex 2 (PRC2). It regulates the expression of a broad range of genes and thereby controls a variety of basic cellular functions [8]. In more detail, EZH2 serves as histone methyltransferase that catalyzes trimethylation of lysine 27 of histone H3 (H3K27me3), which in turn causes the transcriptional repression of its target genes. Inactivation of $E Z H 2$ ( $\left.E Z H 2^{\text {inact }}\right)$-either by mutation, deletion or a decrease in $E Z H 2$ expression-can be

6 Gottfried Schatz Research Center for Cell Signaling, Metabolism \& Aging, Medical University of Graz, Graz, Austria

7 Division of Cell Biology, Histology and Embryology, Medical University of Graz, Graz, Austria

8 BioTechMed-Graz, Graz, Austria

9 University Hospital Heidelberg, Heidelberg, Germany

10 Diagnostic and Research Institute of Pathology, Medical University of Graz, Graz, Austria

11 MRC Cancer Unit, University of Cambridge, Hutchison/MRC Research Centre, Cambridge, United Kingdom

12 Otto-Loewi Research Center for Vascular Biology, Immunology and Inflammation, Division of Pharmacology, Medical University of Graz, Graz, Austria 
observed in a series of MN [8, 9]. Recently, EZH2 $2^{\text {inact }}$ has been linked to RAS-signaling as Ezh2 deletion aggravated the development of Nras $^{\text {mut }}$-driven MN in mice. Moreover, Ezh2 deletion in Kras ${ }^{\text {mut }}$-induced lung cancers hyperactivated $\mathrm{Kras}^{\text {mut }}$-driven MAPK/ERK-signaling [10]. These findings suggest that the dependence on activated RASsignaling in $R A S^{\text {mut }}$ tumors might be altered by the additional occurrence of $E Z H 2^{\text {inact }}$.

In this study, we aimed to investigate this hypothesis in the context of myeloid leukemogenesis. By studying almost 450 primary patient specimens with chronic myelomonocytic leukemia (CMML) and acute myeloid leukemia (AML), we show that $E Z H 2^{\text {inact }}$ and $R A S^{\text {mut }}$ co-exist in MN, and that this co-occurrence is associated with a poor prognosis in affected patients. Importantly, however, we further demonstrate that concomitant $E Z H 2^{\text {inact }}$ and $R A S^{\text {mut }}$ increases the dependence on RAS-signaling and, consequently, the sensitivity to pharmacologic MEK inhibition in myeloid leukemia cells.

Initially, we were interested whether $E Z H 2^{\text {inact }}$ and $R A S^{\text {mut }}$ indeed co-exist in MN. Therefore, we re-analyzed previously published Next-Generation Sequencing (NGS) data of 260 chronic myelomonocytic leukemia (CMML) patients within the Austrian Biodatabase for CMML [11]. We chose this entity, since CMML is often driven by mutations modifying the $R A S$ genes $[1,3,6]$. Within this cohort, $112 / 260(43.1 \%)$ patients exhibited at least one $R A S^{m u t}, E Z H 2$ was mutated in 50/260 (19.2\%) cases and 32/ $260(12.3 \%)$ presented with both genetic aberrations together. 32/112 (28.6\%) RAS ${ }^{\text {mut }}$ patients exhibited additional EZH2 mutations, whereas 32/50 (64\%) cases with EZH2 mutations presented with an additional $R A S^{\text {mut }}$ (Fig. 1A; Supplementary Table 1). Importantly, the frequency of patients with $E Z H 2$ mutations was increased in cases with one or more $R A S^{\text {mut }}$ (28.6\% in $R A S^{\text {mut }}$, vs. $12.2 \%$ in $R A S^{w t}$; $P=0.001$; Fig. 1B). From a clinical point of view, $R A S^{m u t}$ and $E Z H 2$ aberration co-occurrence was associated with a shortened overall survival (median 14 vs 29 months, $P=$ 0.005; Fig. 1C). To delineate whether these findings are of relevance for other $\mathrm{MN}$ as well, we then performed a database retrieval of 187 AML patients via The Cancer Genome Atlas (TCGA) (see supplementary methods for details) [12]. In addition to clinical parameters, this database comprises information about mutations, gene expression and DNA copy number variations [12]. Out of the 187 patients within this cohort, $33(17.6 \%)$ exhibited at least one $R A S^{m u t}, 25 / 187$ (13.4\%) exhibited inactivation of $E Z H 2$ and 9/187 (4.8\%) presented with both genetic aberrations together. $9 / 33(27.3 \%) R A S^{m u t}$ patients exhibited with additional $E Z H 2^{\text {inact }}$, whereas $9 / 25$ (36\%) cases with $E Z H 2^{\text {inact }}$ presented with an additional $R A S^{\text {mut }}$ (Fig. 1A; Supplementary Table 2). Moreover, in line with our data from CMML, EZH2 $2^{\text {inact }}$ was significantly more common in
$R A S^{m u t}$ cases $\left(27.3 \%\right.$ in $R A S^{m u t}$ vs. $10.4 \%$ in $R A S^{w t} ; P=$ 0.020; Fig. 1B; EZH2 $2^{\text {inact }}$ defined as $E Z H 2$ mutations and/or copy number losses). As in CMML, this genetic coexistence was associated with a dismal outcome (median survival 7 vs 19 months, $P=0.039$, Fig. 1C). Accordingly, the mRNA expression of $E Z H 2$ was significantly decreased in AML cases carrying one or more $R A S^{m u t}$ (Supplementary Fig. 1). Taken together, these data indicate that $R A S^{\text {mut }}$ and EZH2 aberrations indeed co-exist in human MN and that this co-occurrence seems to be associated with a poor prognosis. Hence, novel therapeutic approaches are desperately needed for these patients, particularly as $R A S^{\text {mut }}$ has been described as difficult to target so far.

Next, we investigated whether $E Z H 2^{\text {inact }}$ influences the $R A S^{\text {mut }}$-driven MAPK/ERK activation in myeloid leukemia cells (for details on materials and methods see supplementary data). For this purpose, we chose two myeloid cell lines (HL-60 and THP-1). Both carry an activating $R A S^{m u t}$, show normal $E Z H 2$ mRNA expression and lack other EZH2 aberrations (Supplementary Fig. 2 and Supplementary Table 3). We treated these cells with the two EZH2 inhibitors GSK-126 and 3-Deazaneplanocin A (DZNep), respectively. While GSK-126 is an enzymatic inhibitor, which does not affect EZH2 protein expression itself, DZNep induces EZH2 protein degradation [9]. Both drugs successfully inhibited EZH2 activity, as assessed by reduced H3K27me3 levels. Importantly, however, both inhibitors caused hyperactivation of RAS-MAPK/ERK-signaling, as evidenced by increased phosphorylation of ERK (pERK; Fig. 2A, B; Supplementary Fig. 3A, B). To exclude potential unspecific off-target effects of the EZH2 inhibitors used, we established a puromycin-selected stable short hairpin RNA (shRNA)-mediated EZH2-knockdown (EZH2-KD) after lentiviral transduction in both cell lines. Empty vectortransduced cells served as controls. Again, EZH2-KD reduced $\mathrm{H} 3 \mathrm{~K} 27 \mathrm{me} 3$ levels and simultaneously increased pERK (Fig. 2C, Supplementary Fig. 4), which indicates that $E Z H 2^{\text {inact }}$ amplifies MAPK/ERK activation in $R A S^{\text {mut }}$ myeloid cells. Next, we explored whether $E Z H 2^{\text {inact }}$ increases the sensitivity to MEK inhibitors in $R A S^{m u t}$ myeloid cells. Therefore, we treated HL-60 and THP-1 cells with and without EZH2-KD with the MEK inhibitor U0126. U0126 efficiently inhibited pERK in all conditions tested. Most importantly, however, the U0126-induced apoptosis was significantly increased in cells with additional EZH2-KD (Fig. 2D; Supplementary Fig. 5), which indicates that these cells are hypersensitive to pharmacologic inhibition of the MAPK/ERK pathway. These findings could be corroborated in 7-AAD/BrdU cell cycle/proliferation assays. Again, the U0126-mediated decrease in proliferation was enhanced in cells with additional EZH2-KD (Supplementary Fig. 6). We then aimed to shed more light on the mechanisms behind $E Z H 2^{\text {inact }}$-induced MAPK-hyperactivation in $R A S^{\text {mut }}$ 
A
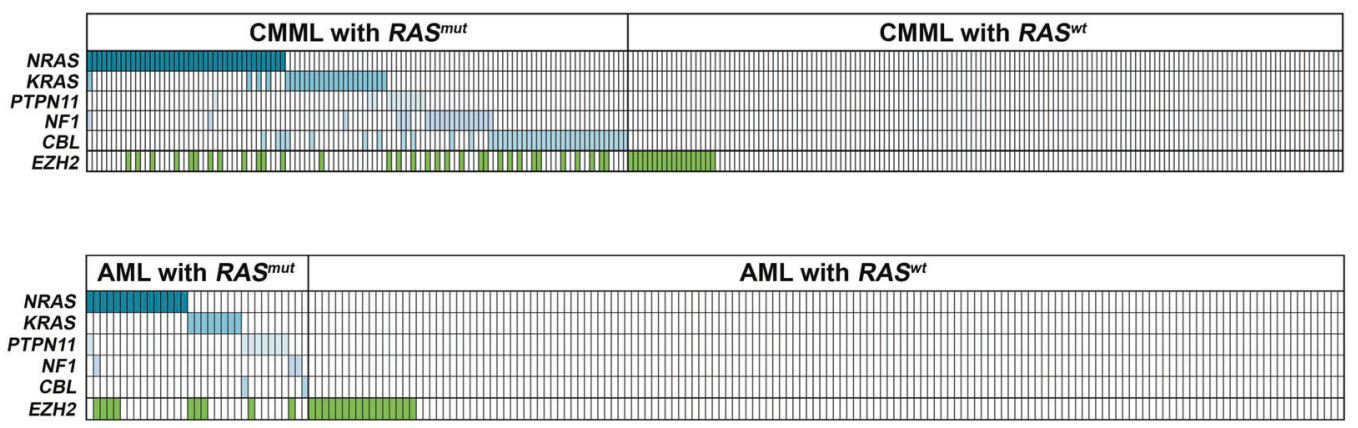

B
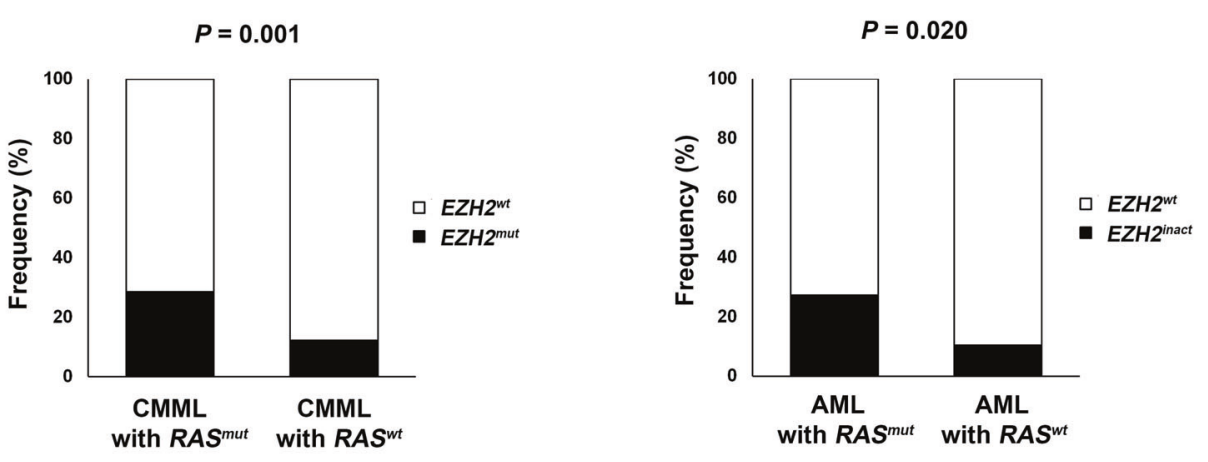

C

CMML patients

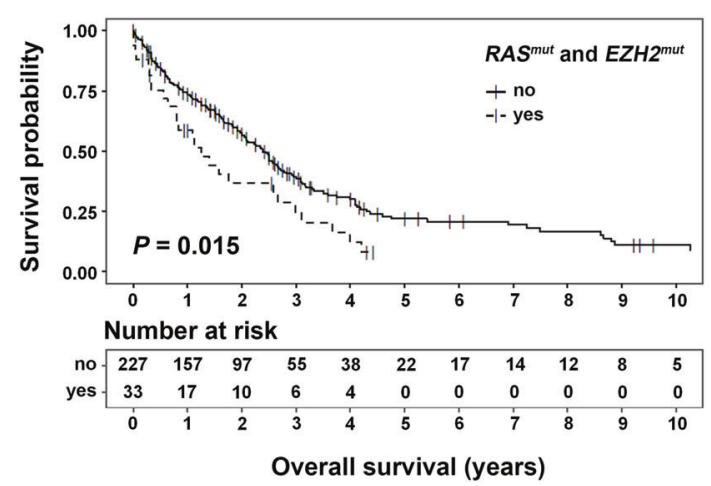

Fig. 1 Association between mutations modifying RAS and EZH2 aberrations in MN. A Next-Generation Sequencing (NGS) results of 260 chronic myelomonocytic leukemia (CMML) patients studied within the Austrian Biodatabase for CMML [15] showing the distribution of mutations modifying $R A S$ ( $R A S^{\text {mut }}$; defined as mutations in $K R A S$ and $N R A S$, as well as in the RAS-GTP modulators NF1, PTPN11 and $C B L$ ) and EZH2. In summary, 112/260 (43.1\%) and 50/ $260(19.2 \%)$ CMML patients had one or more $R A S^{\text {mut }}$ or EZH2 mutation(s) $\left(E Z H 2^{m u t}\right)$, respectively. Below are the results of the database retrieval of 187 acute myeloid leukemia (AML) patients via The Cancer Genome Atlas (TCGA) [12] showing the distribution of $R A S^{\text {mut }}$ and $E Z H 2$ inactivation $\left(E Z H 2^{\text {inact }}\right.$; defined as $E Z H 2$ mutations and/or copy number losses). Every column describes one CMML or AML patient specimen. Colored fields indicate the presence of at least

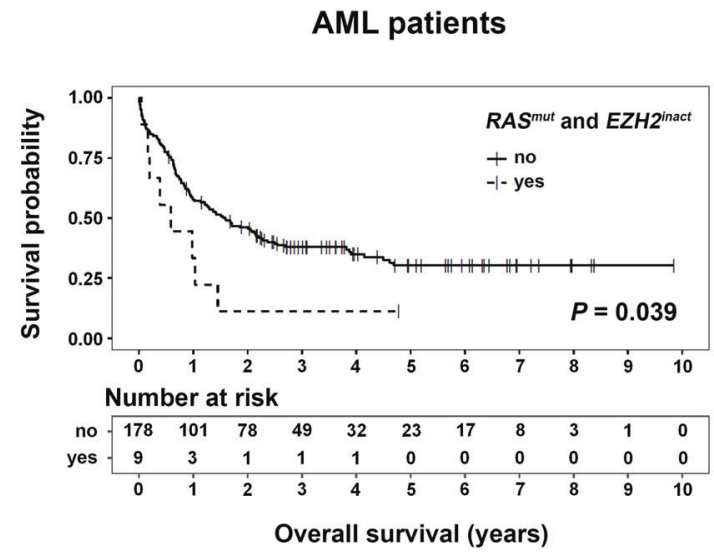

one mutation (for $R A S^{\text {mut }}$ ) or $E Z H 2^{\text {inact }}$, respectively. In summary, 33/ $187(17.6 \%)$ and 25/187 (13.4\%) AML patients had one or more $R A S^{\text {mut }}$ mutation(s) or inactivation of $E Z H 2$, respectively. B Within both cohorts, EZH2 aberrations were significantly more common in patients harboring one or more $R A S^{m u t}$ compared to those without: $28.6 \%$, vs. $12.2 \%(P=0.001)$ for the CMML cohort (left), and $27.3 \%$, vs. $10.4 \%(P=0.020)$ for the AML cohort (right). Fisher's exact test was employed for the statistical analysis. C Survival curves of the patients belonging to the CMML cohort (left), and the TCGA AML cohort (right). In both cohorts, $R A S^{\text {mut }}$ and EZH2 aberration cooccurrence was associated with a shortened overall survival (median 14 vs 29 months and 7 vs 19 months for the CMML and AML patients, respectively). Censored events are indicated by a vertical line. A log-rank test was used for these comparisons. 
A

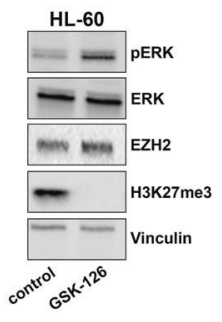

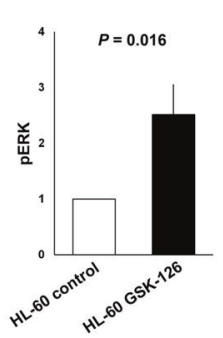

C

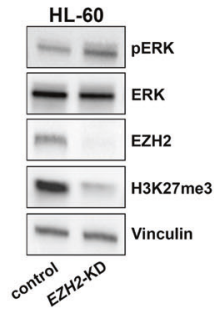

D

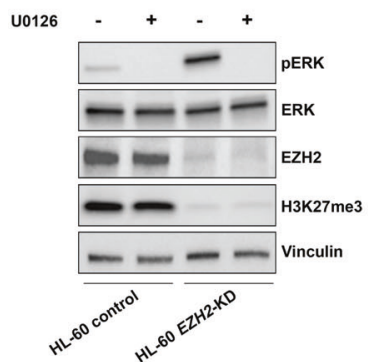

E
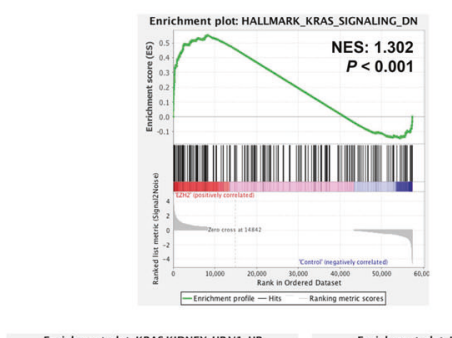

B
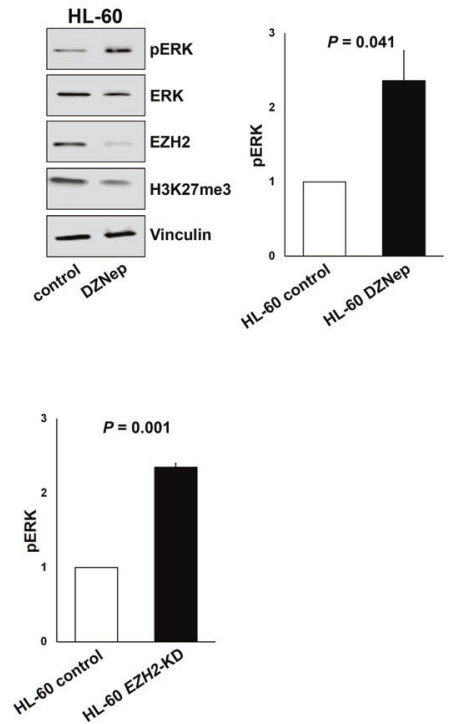
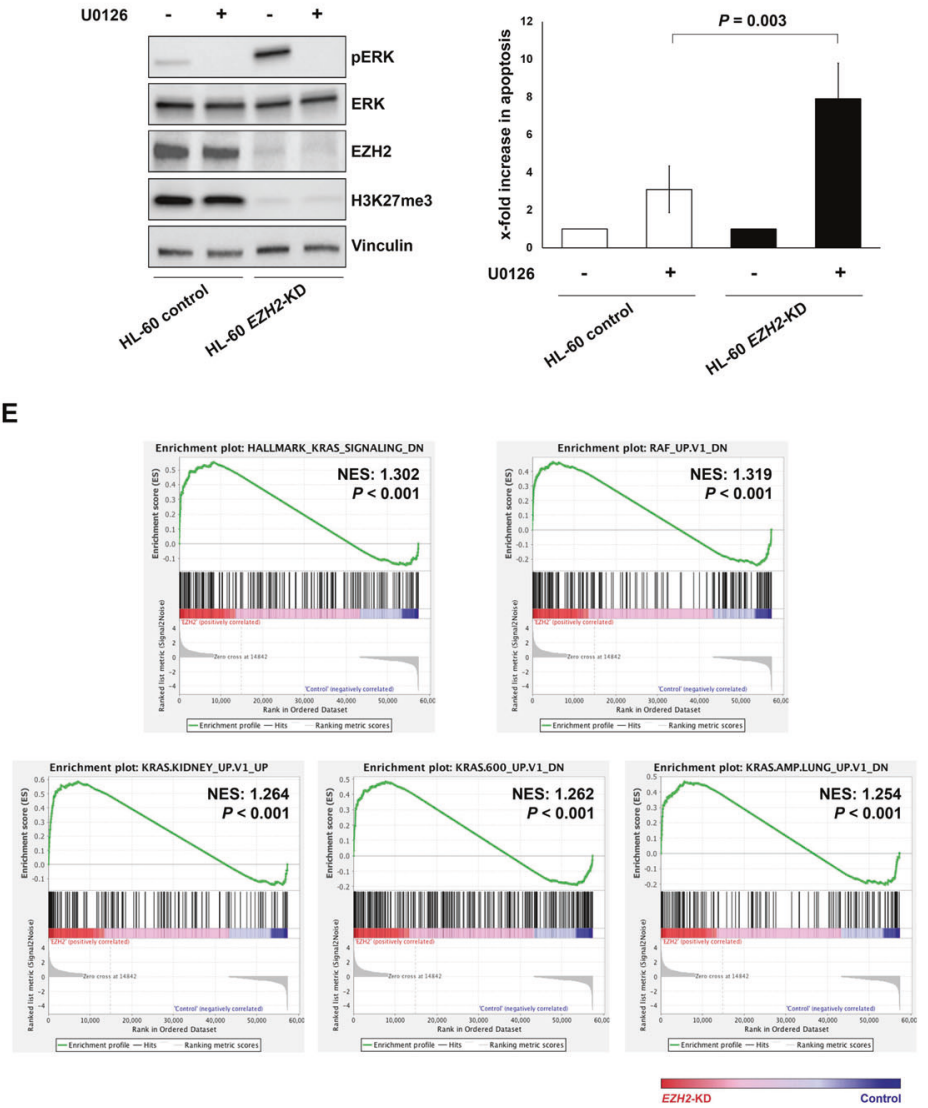

myeloid cells. As EZH2 regulates a multitude of cellular gene expression profiles via H3K27me3-induced transcriptional repression, we reasoned that $E Z H 2^{\text {inact }}$ causes the upregulation of genes involved in RAS-MAPK/ERK-signaling. Such a scenario has been identified in a murine invivo model of $\mathrm{Kras}^{\mathrm{mut}} / \mathrm{Ezh} 2$-deleted lung cancer previously
[10]. To test this assumption, we performed RNAsequencing in HL-60 cells with and without EZH2-KD and performed gene set enrichment analysis (GSEA) [13, 14]. Indeed, we observed enrichment of RAS- and RAF-signaling signatures in the EZH2-KD situation (Fig. 2E). This included an extensive list of genes that 
Fig. 2 EZH2 inactivation in $R A S^{m u t}$ myeloid cells amplifies MAPK/ ERK-signaling and drives MEK inhibitor sensitivity. The activation of the MAPK/ERK pathway was assessed by the phosphorylation of ERK (pERK) by Immunoblot in HL-60 cells (NRAS Q61L-mutated) after treatment with the EZH2 inhibitors GSK-126 (A) and DZNep (B). GSK-126 was added at a concentration of $3 \mu \mathrm{M}$ for 7 days, DZNep at a concentration of $2 \mu \mathrm{M}$ for $24 \mathrm{~h}$. C These experiments were repeated after lentiviral shRNA-mediated $E Z H 2$ knockdown (EZH2$\mathrm{KD})$. The graphs denote the relative increase of pERK expression in the EZH2 inhibitor/KD conditions compared to controls and represent the mean \pm standard deviation (SD) of at least three independent experiments. Comparisons against the control condition were performed using a one-sample $t$ test against a reference value of 1 . D HL60 cells with and without $E Z H 2-\mathrm{KD}$ were treated with the MEK inhibitor U0126 (5 $\mu \mathrm{M}$ for $24 \mathrm{~h}$ ). Subsequently, pERK was assessed by Immunoblot and apoptosis was measured by Annexin-V/7-AAD assay. The graphs denote the $\mathrm{x}$-fold increase in apoptosis in U0126treated cells compared to the respective vehicle-treated control situation in at least three independent experiments and represent the mean \pm SD. Differences between cells with and without EZH2-KD were assessed by paired $t$ test. E Gene set enrichment analysis (GSEA) demonstrating that signatures associated with RAS- and RAFsignaling are enriched within the EZH2-KD situation. All signatures displayed exhibited a false discovery rate of below $25 \%$. NES, normalized enrichment score.

activate the RAS-MAPK/ERK and other signal transduction cascades (Supplementary Table 4).

Finally, we re-analyzed a previously published ChIP-seq dataset of AML cells with EZH2 loss [9] via the NCBI Gene Expression Omnibus (GSE61785). By focusing on genes with a well-described activator function of RAS-signaling on the one hand, and a significant upregulation in our RNAseq data of $E Z H-K D$ cells on the other hand, we were able to demonstrate decreased H3K27me3 signals in the condition with EZH2 loss (Supplementary Fig. 7). These data suggest that the upregulation of these genes in $E Z H 2^{\text {inact }}$ cells could indeed be mediated through modification of H3K27me3 within their promoter and/or adjacent genomic regions.

In conclusion, we demonstrate that mutations within genes modifying $R A S$ frequently co-occur with inactivation of the epigenetic modifier EZH2 in MN, and that this coexistence is linked to a dismal outcome in affected patients. We further demonstrate that inactivation of $E Z H 2$ amplifies the activation of RAS-MAPK/ERK-signaling in myeloid cells carrying $R A S$-modifying mutations. Most importantly, however, we present preclinical data showing that the coexistence of $E Z H 2$ inactivation and $R A S$-modifying mutations might confer increased sensitivity to MEK inhibitors, thereby providing a potential novel therapeutic rationale for these difficult to treat patients.

Acknowledgements We are thankful to all study participants for sample donation. We would also like to acknowledge the TCGA Research Network (http://cancergenome.nih.gov/) for generating datasets analyzed within this article. In this respect, the authors are also thankful to the contribution of the appropriate specimen donors and research groups. This project was supported by Biobank Graz. This study was supported by research funding from the Austrian Society of Internal Medicine (Joseph-Skoda Awards to AZ and AR) and the Austrian Science Fund (grants P 31430-458 B26 to HS and P32783 to $\mathrm{AR})$. Research in the laboratories of AZ, AW, and HS is further supported by Leukämiehilfe Steiermark. AR was also supported by the Austrian Society of Hematology and Medical Oncology (Clinical Research Grant) and MEFOgraz. PhD candidate JL Berg received funding from the MUG within the PhD program Molecular Medicine.

Author contributions JLB, BP, SH, BU, TP, MS, KK, and VC performed the research; KG, CMT, AW, HS, and AZ contributed essential reagents, tools or patient specimens; JLB, AR, MS, KG, CMT, GH, AP, AW, HS, VC, and AZ analyzed the data; GP, GB, and MS performed the statistical analyzes; $\mathrm{VC}$ and $\mathrm{AZ}$ wrote the manuscript; VC and AZ designed the research study; all authors read the manuscript and/or revised it critically; all authors approved the submitted and final version.

\section{Compliance with ethical standards}

Conflict of interest The authors declare no competing interests.

Publisher's note Springer Nature remains neutral with regard to jurisdictional claims in published maps and institutional affiliations.

Open Access This article is licensed under a Creative Commons Attribution 4.0 International License, which permits use, sharing, adaptation, distribution and reproduction in any medium or format, as long as you give appropriate credit to the original author(s) and the source, provide a link to the Creative Commons license, and indicate if changes were made. The images or other third party material in this article are included in the article's Creative Commons license, unless indicated otherwise in a credit line to the material. If material is not included in the article's Creative Commons license and your intended use is not permitted by statutory regulation or exceeds the permitted use, you will need to obtain permission directly from the copyright holder. To view a copy of this license, visit http://creativecommons. org/licenses/by/4.0/.

\section{References}

1. Patnaik MM, Tefferi A. Cytogenetic and molecular abnormalities in chronic myelomonocytic leukemia. Blood Cancer J. 2016;6: e393.

2. Wandler A, Shannon K Mechanistic and Preclinical Insights from Mouse Models of Hematologic Cancer Characterized by Hyperactive Ras. Cold Spring Harb Perspect Med. 2018; 8: https://doi. org/10.1101/cshperspect.a031526.

3. Akutagawa J, Huang TQ, Epstein I, Chang T, QuirindongoCrespo M, Cottonham CL, et al. Targeting the PI3K/Akt pathway in murine MDS/MPN driven by hyperactive Ras. Leukemia. 2016;30:1335-43.

4. Zebisch A, Czernilofsky AP, Keri G, Smigelskaite J, Sill H, Troppmair J. Signaling through RAS-RAF-MEK-ERK: from basics to bedside. Curr Med Chem. 2007;14:601-23.

5. Smith CC, Shah NP. The role of kinase inhibitors in the treatment of patients with acute myeloid leukemia. Am Soc Clin Oncol Educ Book. 2013;33:313-8

6. Caraffini V, Geiger O, Rosenberger A, Hatzl S, Perfler B, Berg JL, et al. Loss of RAF kinase inhibitor protein is involved in myelomonocytic differentiation and aggravates RAS-driven myeloid leukemogenesis. Haematologica. 2020;105:375-86. 
7. Kunimoto H, Meydan C, Nazir A, Whitfield J, Shank K, Rapaport $\mathrm{F}$, et al. Cooperative epigenetic remodeling by TET2 loss and NRAS mutation drives myeloid transformation and MEK inhibitor sensitivity. Cancer Cell. 2018;33:44,59.e8.

8. Rinke J, Chase A, Cross NCP, Hochhaus A, Ernst T. EZH2 in myeloid malignancies. Cells. 2020;9:E1639 https://doi.org/10. 3390/cells9071639.

9. Gollner S, Oellerich T, Agrawal-Singh S, Schenk T, Klein HU, Rohde $\mathrm{C}$, et al. Loss of the histone methyltransferase EZH2 induces resistance to multiple drugs in acute myeloid leukemia. Nat Med. 2017;23:69-78.

10. Wang Y, Hou N, Cheng X, Zhang J, Tan X, Zhang C, et al. Ezh2 acts as a tumor suppressor in Kras-driven lung adenocarcinoma. Int J Biol Sci. 2017;13:652-9.

11. Geissler K, Jager E, Barna A, Gurbisz M, Graf T, Graf E, et al. Correlation of RAS-Pathway Mutations and Spontaneous Myeloid Colony Growth with Progression and Transformation in Chronic Myelomonocytic Leukemia-A Retrospective Analysis in 337
Patients. Int J Mol Sci. 2020;21: https://doi.org/10.3390/ ijms21083025.

12. Cancer Genome Atlas Research Network. Genomic and epigenomic landscapes of adult de novo acute myeloid leukemia. $\mathrm{N}$ Engl J Med. 2013;368:2059-74.

13. Mootha VK, Lindgren CM, Eriksson KF, Subramanian A, Sihag $\mathrm{S}$, Lehar J, et al. PGC-1alpha-responsive genes involved in oxidative phosphorylation are coordinately downregulated in human diabetes. Nat Genet. 2003;34:267-73.

14. Subramanian A, Tamayo P, Mootha VK, Mukherjee S, Ebert BL, Gillette MA, et al. Gene set enrichment analysis: a knowledgebased approach for interpreting genome-wide expression profiles. Proc Natl Acad Sci USA. 2005;102:15545-50.

15. Geissler K, Jager E, Barna A, Gurbisz M, Marschon R, Graf T, et al. The Austrian biodatabase for chronic myelomonocytic leukemia (ABCMML): a representative and useful real-life data source for further biomedical research. Wien Klin Wochenschr. $2019 ; 131: 410-8$ 